Supporting Information

\title{
A General Acid-Base Mechanism for the Stabilization of Tetrahedral Adduct in a Serine-Carboxyl Peptidase: a Computational Study
}

\author{
Haobo $\mathrm{Guo}^{\dagger}$, Alexander Wloderwer ${ }^{\ddagger}$, and Hong Guo*, ${ }^{\dagger}$ \\ ${ }^{\dagger}$ Department of Biochemistry and Cellular and Molecular Biology, Center of Excellence in \\ Structural Biology, University of Tennessee, Knoxville, Tennessee 37996-0840 and ${ }^{\ddagger}$ Protein \\ Structure Section, Macromolecular Crystallography Laboratory, National Cancer Institute at \\ Frederick, Frederick, MD 21702
}

Contents:

- Computational methods;

- Additional structure figures: Figure S1 S3

- Complete Ref. 10 


\section{Computational Method}

A fast semi-empirical density-functional approach (SCC-DFTB $)^{8}$ recently implemented in the CHARMM program ${ }^{9}$ was used for quantum mechanical/molecule mechanical (QM/MM) molecular dynamics (MD) and free energy (potential of mean force or PMF) simulations. The initial coordinates for the simulations were obtained from the crystal structure (PDB ID:1SIO. Resolution: $1.80 \AA$ ) of kumamolisin- $A s$ complexed with the inhibitor AcIPF ( $N$-acetyl-isoleucylprolyl-phenylanial) that is covelantly bound to Ser278. ${ }^{\text {a }}$

The stochastic boundary molecular dynamics method ${ }^{12}$ was used for QM(SCC-DFTB)/MM simulations. The system was separated into a reaction zone and a reservoir region; the reaction zone was further divided into the reaction region and buffer region. The reaction region was a sphere with radius $R$ of $16 \AA$, and the buffer region had $\mathrm{R}$ equal to $16 \AA \leqslant \mathrm{R} \leqslant 18 \AA$. The reference center for partitioning the system was chosen to be the $\mathrm{O}_{\gamma}$ atom of Ser278 (see figure 1A). The resulting system contains 3453 atoms (2700 enzyme atoms and 251 water molecules). The inhibitor AcIPF and the side chains of Glu32, Asp82, Glu78, Ser278 and Asp164 were treated by QM (a total of 92 QM atoms) and the rest of the system by MM. The all-hydrogen potential function (PARAM22) ${ }^{5}$ was used for MM atoms. The link-atom approach (Reuter, N.; Dejaegere, A.; Maigret, B.; Karplus, M. J. Phys. Chem. A 2000, 104, 1720-1735) was used to separate the QM and MM regions. A modified TIP3P water model ${ }^{11}$ was employed for the solvent. The initial structure for the entire stochastic boundary system was optimized by adopted basis Newton-Rhaphson (ABNR) method. The system was gradually heated from 50 to $310 \mathrm{~K}$ for $30 \mathrm{ps}$, and equilibrated at $310 \mathrm{~K}$ for $40 \mathrm{ps}$. A 1-fs time step was used for integration of the equations of motion, and coordinates were saved every $10 \mathrm{fs}$ for analyses. The LD frictional constants were $250 \mathrm{ps}^{-1}$ for the protein atoms and $62 \mathrm{ps}^{-1}$ for the water molecules. The bonds involving hydrogen atoms in the MM region were fixed by the SHAKE algorithm (Ryckaert, J. P.; Ciccotti, G.; Berendsen, H. J. C. J. Comp. Phys. 1977, 23, 327-341). Energy minimization at the B3LYP/6-31G**/MM level was performed to compare with results from the QM(SCCDFTB)/MM calculations (see Figure S1).

The umbrella sampling method ${ }^{13}$ implemented in the CHARMM program along with the Weighted Histogram Analysis Method (WHAM) ${ }^{14}$ was applied to determine the changes of the free energy (potential of mean force). The free energy curves for the wild-type enzyme (with and without the QM treatment of D164), D164N and T277A mutants were obtained as functions of $\xi$ 
$=r\left(\mathrm{C}-\mathrm{O}_{\gamma}\right)$ for the inter-conversion between the tetrahedral adduct and aldehyde complexes. Harmonic biasing potentials were defined by the RESD module of CHARMM, with force constants in range of $100 \sim 800 \mathrm{kcal} \cdot \mathrm{mol}^{-1} \cdot \AA^{-2}$. The free energy curves for the nucleophilic attack and the reverse process were found to be similar (Figure S2). The QM region was enlarged to include W129 or T277 to examine the sensitivity of the free energy profiles on the inclusion of these two additional residues in the QM region. It was found that the results are similar (Figure S2). A further increase of the size of the QM region makes the free energy simulations very expensive, and such simulations were not performed. The systematic and statistical errors (Kobrak, M.N., J. Comput. Chem., 2003, 24, 1437) in the free energy calculations for the wildtype enzyme (i.e., the black solid line in Figure 3A) were determined, and the average error was estimated to be about $\pm 0.2 \mathrm{kca} / \mathrm{mol}$. 
Additional structural figures and 2-D free energy contour for the aldehyde complex

\section{Figure Captions}

Figure S1. Comparison of the average structure from the SCC-DFTB/MM MD simulations with the structure from B3LYP/6-31G** energy minimization (in parentheses). .

Figure S2. The D164N mutant. The average structures for the aldehyde (A) and hemiacetal complexes (B) based on the QM/MM free-energy simulations are given.

Figure S3. The T277A mutant. The average structures for the aldehyde (A) and hemiacetal complexes (B) based on the QM/MM free-energy simulations are given. 


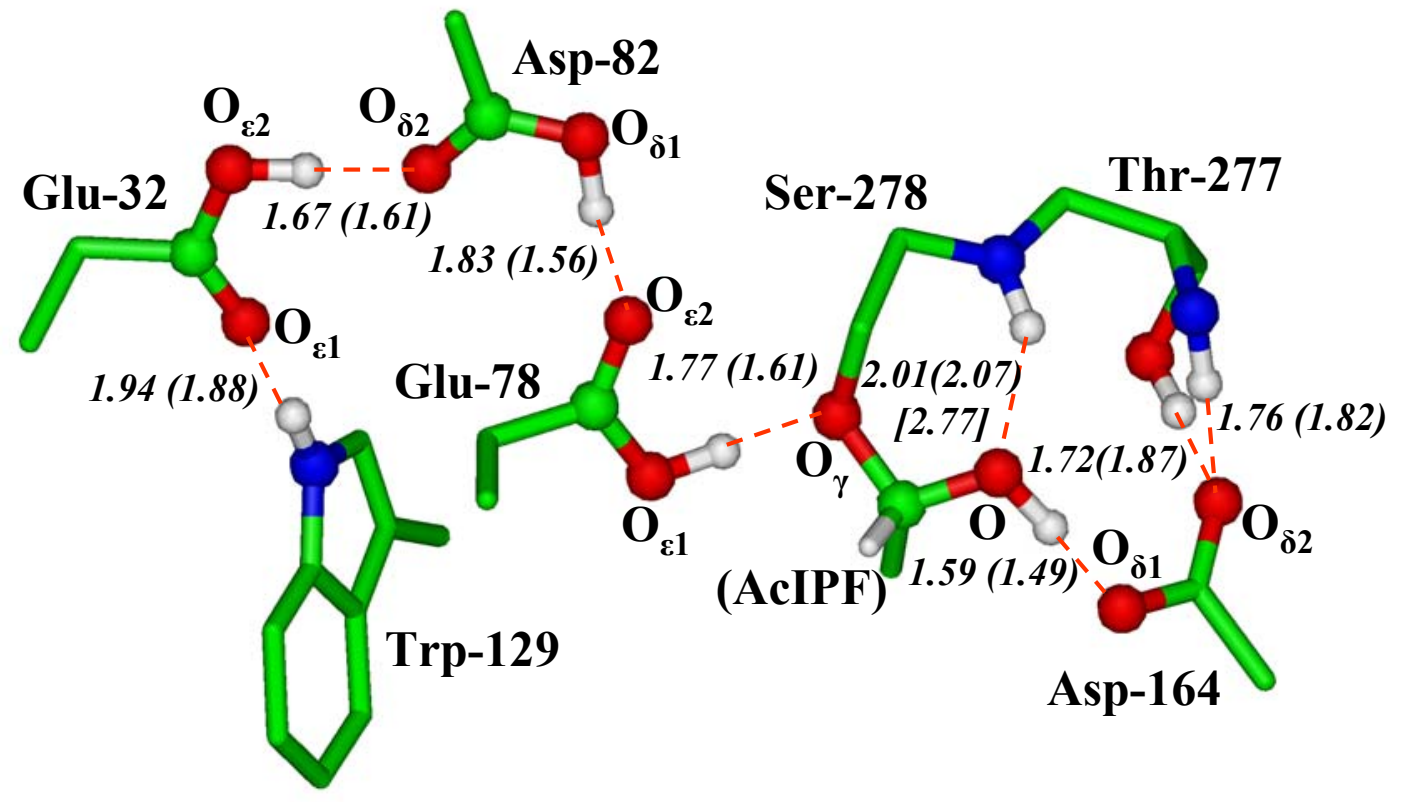

Figure S1 
A

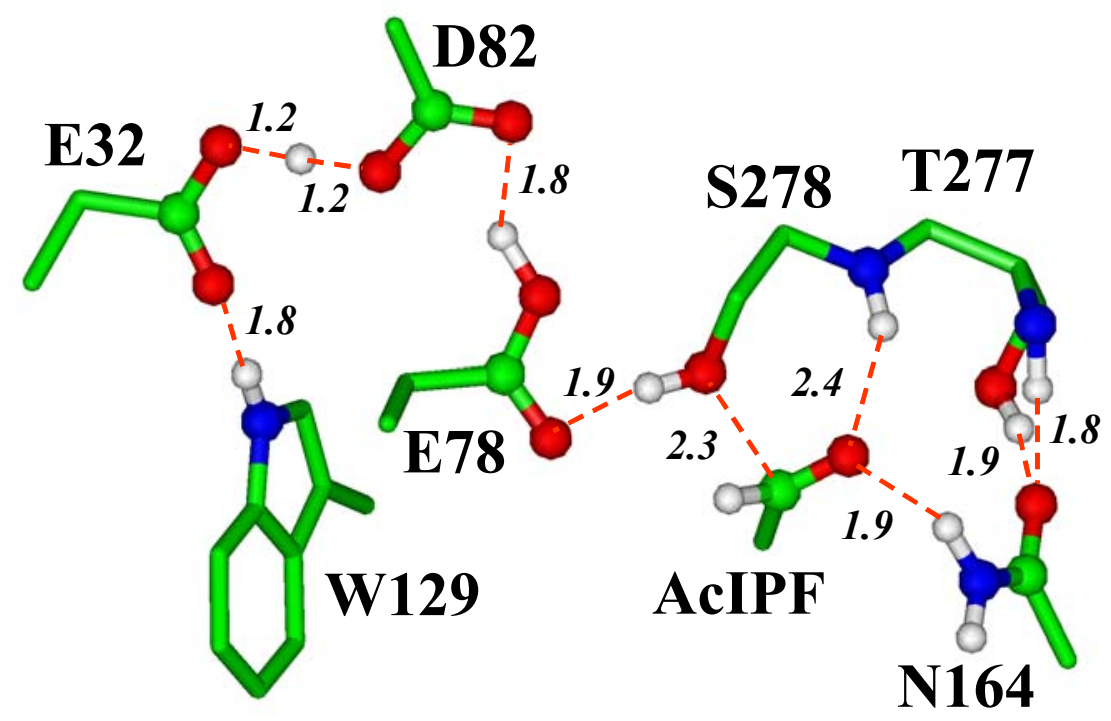

B

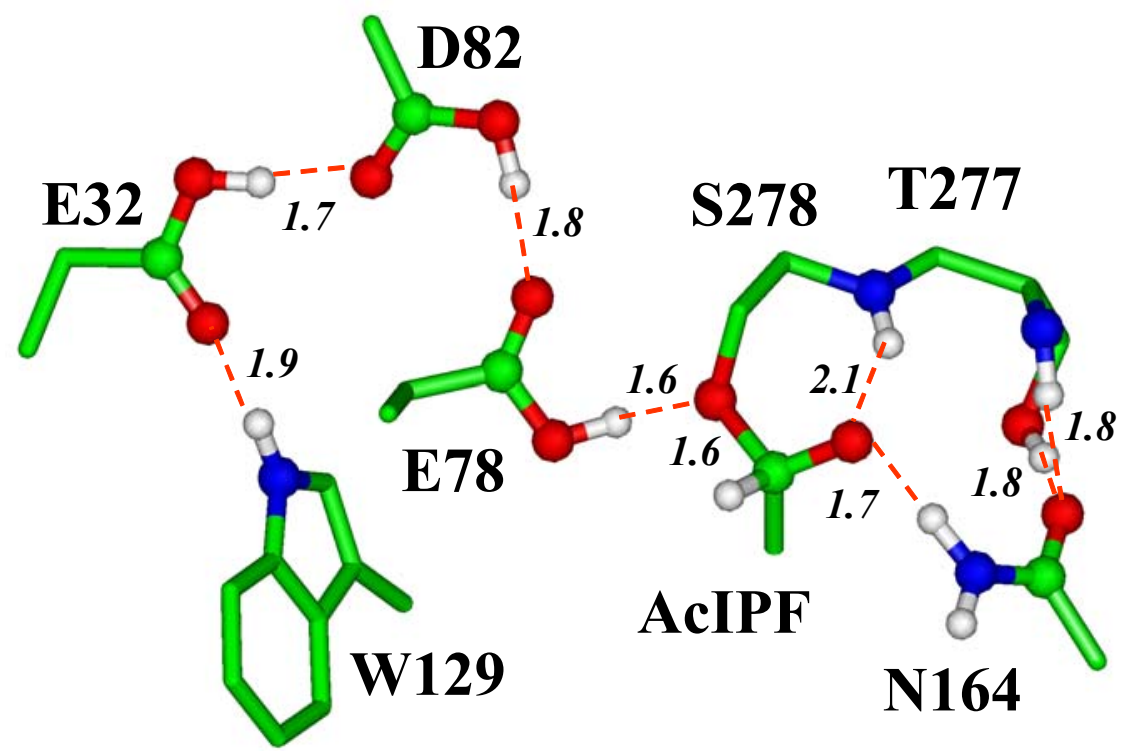

Figure S2 
A

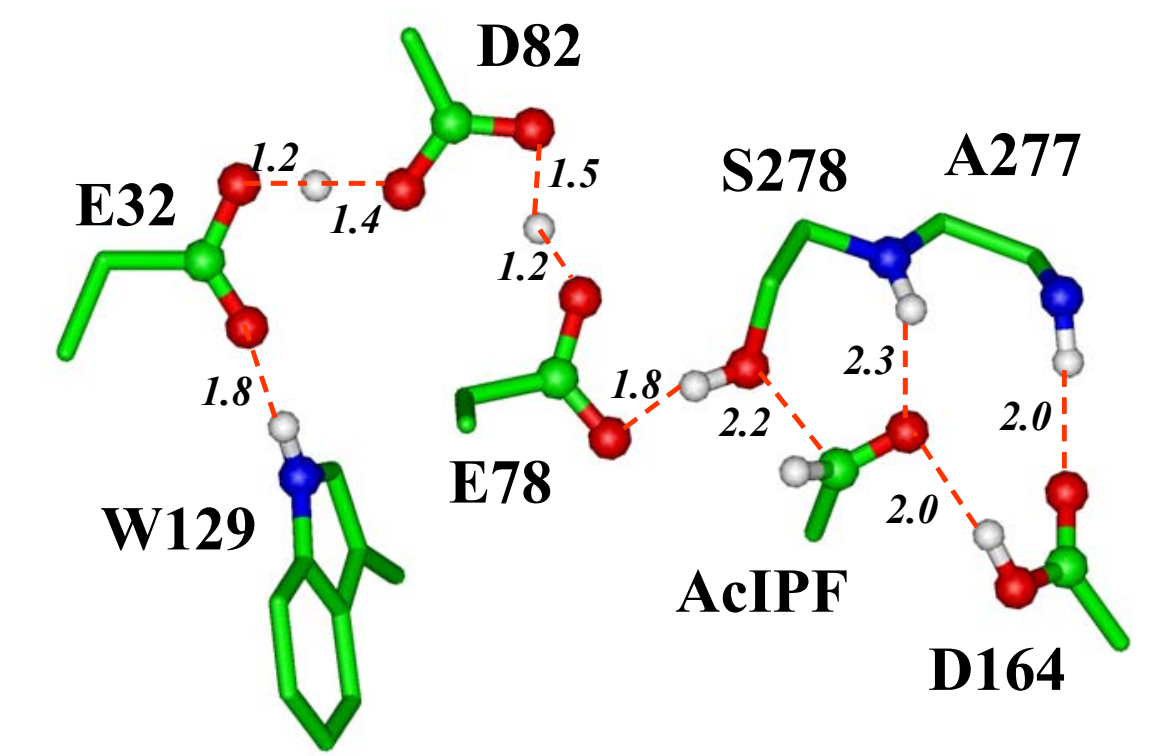

B

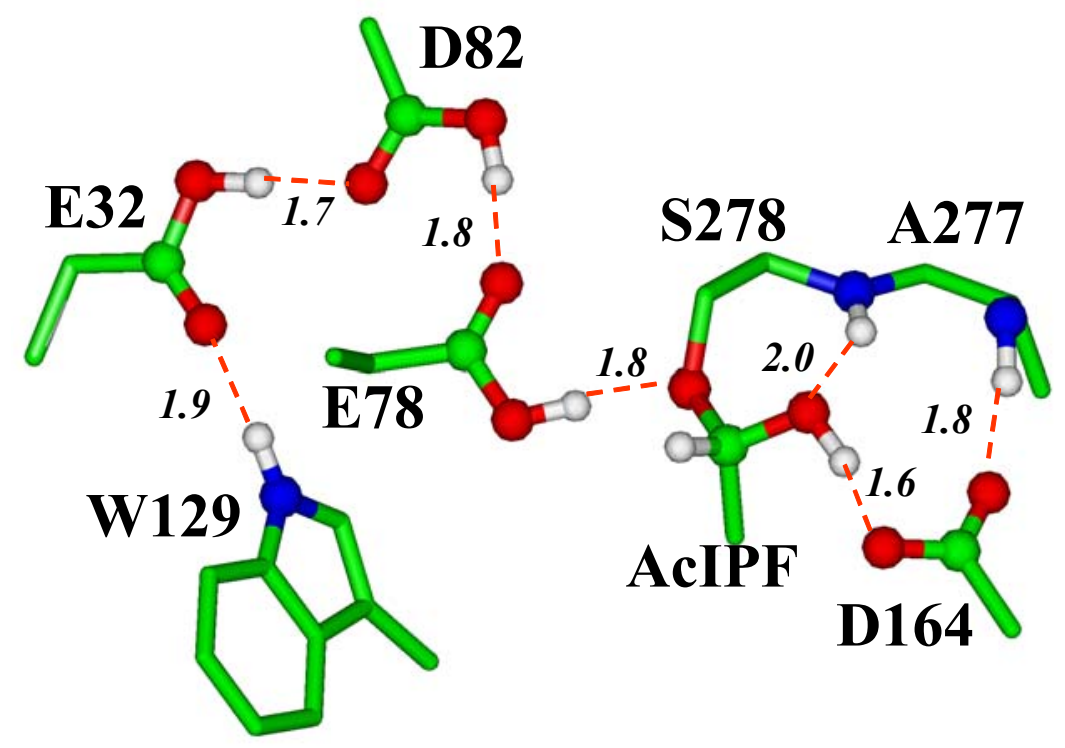

Figure S3 


\section{Complete Ref. 10}

(10). Mackerell, A. D., Jr.; Bashford, D.; Bellott, M.; Bunbrack, R. L., Jr.; Evanseck, J. D.; Field, M. J.; Fischer, S.; Gao, J.; Guo, H.; Ha, S.; Joseph-McCarthy D.; Kuchnir, L.; Kuczera, K.; Lau, F. T. K.; Mattos, C.; Michnick, S.; Ngo, T.; Nguyen, D. T.; Prodhom, B.; Reiher, W. E. III; Roux, B.; Schlenkrich, M.; Smith, J. C.; Stote, R.; Straub, J.; Watanabe, M.; W.-Kuczera, J.; Yin, D.; Karplus, M. J. Phys. Chem. B 1998, 102, 3586-3616. 